\title{
Polypharmacy Associated with Cognitive Decline in Newly Diagnosed Parkinson's Disease: A Cross-Sectional Study
}

\author{
Nobuyuki Ishii Hitoshi Mochizuki Katsuya Sakai Go Ogawa \\ Kazutaka Shiomi Masamitsu Nakazato \\ Division of Neurology, Respirology, Endocrinology and Metabolism, Department of Internal \\ Medicine, University of Miyazaki, Miyazaki, Japan
}

Keywords
Cognitive dysfunction · Mini-Mental State Examination · Parkinson's disease · Polypharmacy

\begin{abstract}
Aims: Polypharmacy is well known to affect cognitive function in community-dwelling older adults. However, the effect of polypharmacy on cognitive function in patients with newly diagnosed Parkinson's disease remains unknown. Here, we evaluated the association between polypharmacy and cognitive function in patients with newly diagnosed Parkinson's disease. Methods: This cross-sectional study enrolled 131 consecutive hospitalized patients with newly diagnosed Parkinson's disease. Cognitive function was evaluated with the Mini-Mental State Examination and analyzed between groups of patients with or without polypharmacy. Comparisons were adjusted for confounders by performing inverse probability weighting with propensity scores. Results: After inverse probability weighting, patients in the polypharmacy group had a significantly lower Mini-Mental State Examination score than patients in the nonpolypharmacy group (26.2 vs. 27.7, $p=0.001$ ). Conclusion: Polypharmacy was associated with cognitive decline in patients with newly diagnosed Parkinson's disease. This finding suggests that medication reduction might serve as a promising intervention to prevent the development of dementia in patients with early Parkinson's disease. Further prospective studies are needed to determine whether medication reduction improves cognitive function in patients with newly diagnosed Parkinson's disease.


Ishii et al.: Polypharmacy Associated with Cognitive Decline in Newly Diagnosed Parkinson's Disease: A Cross-Sectional Study

\section{Introduction}

Polypharmacy is an emerging concern because of its associations with falls, adverse drug events, frailty, hospitalization, and mortality [1]. A recent systematic review found that polypharmacy was related to cognitive impairment in community-dwelling older adults [2].

Patients with Parkinson's disease (PD) are well-known to develop dementia; a prior study showed that $15-20 \%$ of patients with newly diagnosed PD had minimal cognitive impairment (MCI), and $80 \%$ of patients with PD were diagnosed with dementia 20 years after disease onset [3]. MCI is a risk factor for the development of dementia in patients with PD [4]; thus, effective interventions for $\mathrm{MCI}$ in the early stages of PD might prevent the progression of dementia. Medication reduction to avoid polypharmacy is a potential intervention for improvement of cognitive function; however, it remains unknown whether polypharmacy affects cognitive function in patients with newly diagnosed PD. Here, in a cross-sectional study of patients with newly diagnosed PD, we evaluate the association between polypharmacy and cognitive function, as measured with the Mini-Mental State Examination (MMSE) [5].

\section{Materials and Methods}

\section{Patients}

During the period from April 2012 to March 2019, consecutive hospitalized patients who were newly diagnosed with PD at the University of Miyazaki Hospital were enrolled into this cross-sectional study. All of the patients were diagnosed with clinically established or clinically probable PD in accordance with International Parkinson and Movement Disorder Society clinical diagnostic criteria [6]. We excluded patients who could not perform activities of daily living or function independently due to cognitive impairment. Patients were classified into 2 groups according to the presence or absence of polypharmacy, which was defined as treatment with 6 or more medications [7]. The study protocol was approved by the Ethics Committee of the University of Miyazaki. Written informed consent was obtained from all of the participants.

\section{Variables}

We retrospectively collected the following clinical history, comorbidities, and findings associated with PD at the time of diagnosis: MMSE score, age, sex, years of education, history of alcohol intake, smoking, dyslipidemia, diabetes, hypertension, ischemic heart disease, stroke, motor subtype (tremor-dominant, akinetic-rigid, or mixed type) [8], presence of a depressive state, orthostatic hypotension, olfactory dysfunction, rapid eye movement sleep behavior disorder (RBD), Hoehn and Yahr scale [9], and disease duration. Orthostatic hypotension was defined as a reduction of at least $20 \mathrm{~mm} \mathrm{Hg}$ in systolic and/or $10 \mathrm{~mm} \mathrm{Hg}$ in diastolic blood pressure within 3 min of standing [10]. We assessed the presence or absence of symptoms of a depressive state, olfactory dysfunction, and RBD by direct interviews with patients and their families. Years of education were classified as follows, based on the Japanese educational system: 0-9 years, junior high school; 10-12 years, high school; and $\geq 13$ years, post-high school education [7].

\section{Statistical Analysis}

The $t$ test was used to compare continuous variables, while the $\chi^{2}$ test was used to compare categorical variables. Univariable analyses were conducted in the unadjusted patient groups (nonpolypharmacy vs. polypharmacy). The method of inverse probability weighting (IPW) with a propensity score was used to minimize confounding biases. First, a propensity score was constructed using all of the following baseline characteristics (the MMSE score was excluded): age, sex, years of education, social history (alcohol drinking habits and smoking), medical 
Table 1. Clinical characteristics of patients with and without polypharmacy

\begin{tabular}{|c|c|c|c|c|}
\hline & \multirow{2}{*}{$\begin{array}{l}\text { Total } \\
(n=131)\end{array}$} & \multicolumn{3}{|c|}{ Polypharmacy } \\
\hline & & no $(n=88)$ & yes $(n=43)$ & $p$ value \\
\hline MMSE score & $27.1 \pm 2.6$ & $27.8 \pm 2.6$ & $25.8 \pm 2.3$ & $<0.001$ \\
\hline Age, years & $69.8 \pm 8.2$ & $67.7 \pm 7.9$ & $74.3 \pm 6.9$ & $<0.001$ \\
\hline Males & $61(46.6)$ & $41(46.6)$ & $20(46.5)$ & 1.00 \\
\hline Education & & & & 0.008 \\
\hline $0-9$ years & $46(35.1)$ & $23(26.1)$ & $23(53.5)$ & \\
\hline 10-12 years & $59(45.0)$ & $46(52.3)$ & $13(30.2)$ & \\
\hline$\geq 13$ years & $26(19.8)$ & $19(21.6)$ & $7(16.3)$ & \\
\hline Social history & & & & \\
\hline Alcohol drinking habits & $41(31.3)$ & $27(30.7)$ & $14(32.6)$ & 0.99 \\
\hline Smoking & $24(18.3)$ & $14(15.9)$ & $10(23.3)$ & 0.44 \\
\hline \multicolumn{5}{|l|}{ Medical history } \\
\hline Hypertension & $60(45.8)$ & $31(35.2)$ & $29(67.4)$ & 0.001 \\
\hline Diabetes & $19(14.5)$ & $11(12.5)$ & $8(18.6)$ & 0.50 \\
\hline Dyslipidemia & $28(21.4)$ & $16(18.2)$ & $12(27.9)$ & 0.30 \\
\hline IHD & $6(4.6)$ & $1(1.1)$ & $5(11.6)$ & 0.02 \\
\hline Stroke & $8(6.1)$ & $4(4.5)$ & $4(9.3)$ & 0.50 \\
\hline \multicolumn{5}{|l|}{ PD } \\
\hline Disease duration, months & $20.2 \pm 14.7$ & $19.7 \pm 14.7$ & $21.1 \pm 14.7$ & 0.62 \\
\hline Motor subtype & & & & 0.25 \\
\hline Tremor-dominant & $40(30.5)$ & $30(34.1)$ & $10(23.3)$ & \\
\hline Akinetic-rigid & $78(49.5)$ & $48(54.5)$ & $30(69.8)$ & \\
\hline Mixed & $13(9.9)$ & $10(11.4)$ & $3(7.0)$ & \\
\hline Hohen and Yahr scale & & & & 0.08 \\
\hline 1 & $23(17.6)$ & $17(19.3)$ & $6(14.0)$ & \\
\hline 2 & $58(44.3)$ & $43(48.9)$ & $15(34.9)$ & \\
\hline 3 & $39(29.8)$ & $24(27.3)$ & $15(34.9)$ & \\
\hline 4 & $9(6.9)$ & $4(4.5)$ & $5(11.6)$ & \\
\hline 5 & $2(1.5)$ & $0(0)$ & $2(4.7)$ & \\
\hline Depressive state & $19(14.5)$ & $14(15.9)$ & $5(11.6)$ & 0.70 \\
\hline Orthostatic hypotension & $39(29.8)$ & $28(31.8)$ & $11(25.6)$ & 0.60 \\
\hline Olfactory dysfunction & $41(31.3)$ & $30(34.1)$ & $11(25.6)$ & 0.43 \\
\hline RBD & $67(51.1)$ & $39(44.3)$ & $28(65.1)$ & 0.04 \\
\hline
\end{tabular}

Values are presented as means \pm SD or numbers (\%). IHD, ischemic heart disease.

history (hypertension, diabetes, dyslipidemia, ischemic heart disease, and stroke), clinical characteristics of PD (disease duration, motor subtype, Hoehn and Yahr scale, depression, orthostatic hypotension, olfactory dysfunction, and rapid eye movement sleep behavior disorder). IPW with propensity scores was then performed to adjust different covariate distributions for both groups; analyses were then conducted with these IPW-adjusted groups. Statistical analyses were conducted with R software (version 3.5.1; R Foundation for Statistical Computing, Vienna, Austria), and $p<0.05$ was considered statistically significant.

\section{Results}

Table 1 shows the baseline characteristics of the included patients at the time of diagnosis with PD. One hundred thirty-one patients were enrolled into this study. The mean age was $69.8 \pm 8.2$ years, and 61 patients $(46.6 \%)$ were men; 43 patients $(32.8 \%)$ met the criteria 
Ishii et al.: Polypharmacy Associated with Cognitive Decline in Newly Diagnosed Parkinson's Disease: A Cross-Sectional Study

Table 2. MMSE score of the unadjusted and IPW-adjusted groups

\begin{tabular}{|c|c|c|c|c|c|c|}
\hline \multirow[t]{2}{*}{ Group } & \multicolumn{3}{|c|}{ Unadjusted groups } & \multicolumn{3}{|c|}{ IPW-adjusted groups } \\
\hline & no & MMSE & $p$ value & no & MMSE & $p$ value \\
\hline \multicolumn{7}{|c|}{ Polypharmacy } \\
\hline No & 88 & $27.8 \pm 2.6$ & $<0.001$ & 123.5 & $27.7 \pm 2.6$ & 0.001 \\
\hline Yes & 43 & $25.8 \pm 2.3$ & & 108.7 & $26.2 \pm 2.0$ & \\
\hline
\end{tabular}

Values are presented as means \pm SD or numbers. The following confounders were adjusted for IPW with propensity scores: age, sex, years of education, social history (alcohol drinking habits and smoking), medical history (hypertension, diabetes, dyslipidemia, ischemic heart disease, and stroke), clinical characteristics of PD (disease duration, motor subtype, Hohen and Yahr scale, depression, orthostatic hypotension, olfactory dysfunction, and RBD).

for polypharmacy. Univariable analysis using unadjusted patient groups demonstrated significant differences in the following factors: MMSE score, age, years of education, history of hypertension, history of ischemic heart disease, and presence of RBD. The propensity score constructed from the 17 baseline characteristics corresponded well with the data, which showed a receiver operating characteristic curve with a c-statistic of 0.89 . Despite the use of IPW to adjust for confounders, patients in the polypharmacy group continued to exhibit a significantly lower average MMSE score than that of patients in the nonpolypharmacy group (26.2 vs. $27.7, p=0.001$; Table 2 ).

\section{Discussion}

In the present study, we found that patients with newly diagnosed PD who met the criteria for polypharmacy had significantly lower MMSE scores than patients who did not meet the criteria for polypharmacy. After IPW adjustment, patients who met the criteria for polypharmacy had an average MMSE score of 26.2 , which was -1.5 points lower than that of patients who did not meet the criteria for polypharmacy.

Previous studies have assessed the association between polypharmacy and dementia in community-dwelling older adults [2] and found that community-dwelling elderly people who met the criteria for polypharmacy had an average MMSE score that was 0.51 points below that of elderly people who did not meet the criteria for polypharmacy [11]; the difference in MMSE score was greater in our newly diagnosed PD cohort. We speculate that polypharmacy may exhibit a greater effect on the cognitive function of patients with newly diagnosed PD, compared to community-dwelling elderly people, for the following reasons. Generally, people with a relative cerebral functional decline, including older adults or patients with dementia, are likely to develop medication-induced neuropsychiatric symptoms, such as delirium [12]. Indeed, patients with newly diagnosed PD showed reduced cerebral glucose metabolism and cognitive function [13], and 15-20\% of such patients demonstrated MCI [3]. Thus, the combination of polypharmacy with aging and subclinical cerebral dysfunction due to PD pathology may have a greater effect on the cognitive function of patients with newly diagnosed PD compared to community-dwelling elderly people.

We suspect that medication reduction may improve cognitive function in patients with newly diagnosed PD. Some predictive factors for the development of dementia in patients with PD include autonomic dysfunction, RBD, and MCI [4]. Indeed, the median score of MMSE in our 
Ishii et al.: Polypharmacy Associated with Cognitive Decline in Newly Diagnosed

Parkinson's Disease: A Cross-Sectional Study

patients who met the criteria for polypharmacy was $<27$, which was used as the cut-off value for MCI in a previous study [14]; conversely, patients in the present study who did not meet the criteria for polypharmacy had an MMSE score above the cut-off level. Thus, medication reduction might be a means to prevent dementia if it is confirmed to improve cognitive function.

However, the relationship between medication reduction and improved cognitive function is controversial. A previous systematic review found that medication reduction in a general practitioner or family physician setting did not affect clinical outcomes, including cognitive function [15]. In contrast, a study of patients with late-stage PD showed that patients with levodopa-carbidopa intestinal gel infusion therapy (Duodopa ${ }^{\circledR}$ ) had better cognitive function than patients in the polypharmacy group [16]. Medication reduction might have a positive impact on cognitive function in patients with PD. Further studies are needed to confirm this hypothesis in patients with newly diagnosed PD.

There were a few notable limitations in this study. First, this was a cross-sectional study; thus, a few patients with newly diagnosed PD might have later been diagnosed with atypical parkinsonism, such as progressive supranuclear palsy, multiple system atrophy, or corticobasal degeneration. Second, we analyzed the effect of the number of medications without specifically controlling for the use of medications that affect the central nervous system (e.g., benzodiazepines and anticholinergic drugs).

In conclusion, we demonstrated that patients with newly diagnosed PD who met the criteria for polypharmacy had lower cognitive function than patients who did not meet the criteria for polypharmacy. This is the first study to demonstrate an association between polypharmacy and cognitive decline in patients with newly diagnosed PD, as well as in communitydwelling older adults. Medication reduction might be a promising intervention to prevent the development of dementia in patients with early PD. Further prospective studies are needed to confirm whether medication reduction in patients with newly diagnosed PD can improve cognitive function and prevent the development of dementia.

\section{Acknowledgement}

This study was partly supported by JSPS KAKENHI grant No. 19K20715 (N. Ishii). We would like to thank Editage (www.editage.jp) for English language editing.

\section{Statement of Ethics}

The study protocol was approved by the Ethics Committee of the University of Miyazaki. Written informed consent was obtained from all of the participants.

\section{Disclosure Statement}

The authors declare no conflict of interests.

\section{Author Contributions}

N. Ishii: study concept and design; acquisition, analysis, and interpretation of data; statistical analysis; and preparation of this paper. H. Mochizuki: study concept and design; acquisition, analysis, and interpretation of data; and statistical analysis. K. Sakai: acquisition, 
Ishii et al.: Polypharmacy Associated with Cognitive Decline in Newly Diagnosed

Parkinson's Disease: A Cross-Sectional Study

analysis, and interpretation of data; statistical analysis and preparation of this paper. G. Ogawa: acquisition, analysis, and interpretation of data and statistical analysis. K. Shiomi: study concept and design and analysis and interpretation of data. M. Nakazato: study concept and design, analysis and interpretation of data, and statistical analysis. All of the authors critically reviewed this paper.

\section{References}

1 Fried TR, O’Leary J, Towle V, Goldstein MK, Trentalange M, Martin DK. Health outcomes associated with polypharmacy in community-dwelling older adults: a systematic review. J Am Geriatr Soc. 2014 Dec;62(12):226172.

2 Leelakanok N, D'Cunha RR. Association between polypharmacy and dementia: A systematic review and metaanalysis. Aging Ment Health. 2019;23:932-41.

3 Svenningsson P, Westman E, Ballard C, Aarsland D. Cognitive impairment in patients with Parkinson's disease: diagnosis, biomarkers, and treatment. Lancet Neurol. 2012 Aug;11(8):697-707.

4 Anang JB, Gagnon JF, Bertrand JA, Romenets SR, Latreille V, Panisset M, et al. Predictors of dementia in Parkinson disease: a prospective cohort study. Neurology. 2014 Sep;83(14):1253-60.

5 Folstein MF, Folstein SE, McHugh PR. "Mini-mental state”. A practical method for grading the cognitive state of patients for the clinician. J Psychiatr Res. 1975 Nov;12(3):189-98.

6 Postuma RB, Berg D, Stern M, Poewe W, Olanow CW, Oertel W, et al. MDS clinical diagnostic criteria for Parkinson's disease. Mov Disord. 2015 Oct;30(12):1591-601.

7 Niikawa H, Okamura T, Ito K, Ura C, Miyamae F, Sakuma N, et al. Association between polypharmacy and cognitive impairment in an elderly Japanese population residing in an urban community. Geriatr Gerontol Int. 2017 Sep;17(9):1286-93.

8 Spiegel J, Hellwig D, Samnick S, Jost W, Möllers MO, Fassbender K, et al. Striatal FP-CIT uptake differs in the subtypes of early Parkinson's disease. J Neural Transm (Vienna). 2007 Mar;114(3):331-5.

9 Hoehn MM, Yahr MD. Parkinsonism: onset, progression and mortality. Neurology. 1967 May;17(5):427-42.

10 Freeman R, Wieling W, Axelrod FB, Benditt DG, Benarroch E, Biaggioni I, et al. Consensus statement on the definition of orthostatic hypotension, neurally mediated syncope and the postural tachycardia syndrome. Clin Auton Res. 2011 Apr;21(2):69-72.

11 Cheng CM, Chang WH, Chiu YC, Sun Y, Lee HJ, Tang LY, et al. Association of Polypharmacy With Mild Cognitive Impairment and Cognitive Ability: A Nationwide Survey in Taiwan. J Clin Psychiatry. 2018 Sep;79(6):79.

12 Inouye SK, Westendorp RG, Saczynski JS. Delirium in elderly people. Lancet. 2014 Mar;383(9920):911-22.

13 Firbank MJ, Yarnall AJ, Lawson RA, Duncan GW, Khoo TK, Petrides GS, et al. Cerebral glucose metabolism and cognition in newly diagnosed Parkinson's disease: ICICLE-PD study. J Neurol Neurosurg Psychiatry. 2017 Apr; 88(4):310-6.

14 Saxton J, Morrow L, Eschman A, Archer G, Luther J, Zuccolotto A. Computer assessment of mild cognitive impairment. Postgrad Med. 2009 Mar;121(2):177-85.

15 Huiskes VJ, Burger DM, van den Ende CH, van den Bemt BJ. Effectiveness of medication review: a systematic review and meta-analysis of randomized controlled trials. BMC Fam Pract. 2017 Jan;18(1):5.

16 Nyholm D, Nilsson Remahl AI, Dizdar N, Constantinescu R, Holmberg B, Jansson R, et al. Duodenal levodopa infusion monotherapy vs oral polypharmacy in advanced Parkinson disease. Neurology. 2005 Jan;64(2):21623. 